\title{
Especies del género Quercus (Fagaceae) presentes en el área natural protegida de Sierra Fría, Aguascalientes, México
}

\author{
Species of genus Quercus (Fagaceae) in natural protected area of Sierra \\ Fria, Aguascalientes, Mexico
}

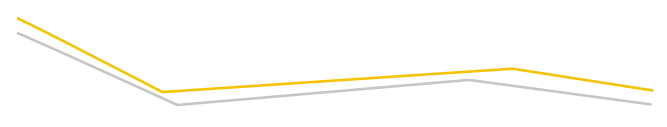

Víctor Manuel Martínez-Calderón*, María Elena Siqueiros-Delgado*®, Julio Martínez-Ramírez*

Martínez-Calderón, V. M., Siqueiros-Delgado, M. E., \& Martínez-Ramírez, J. (2017). Especies del género Quercus (Fagaceae) presentes en el área natural protegida de Sierra Fría, Aguascalientes, México. Investigación y Ciencia de la Universidad Autónoma de Aguascalientes, 25(71), 12-18.

RESUMEN

El género Quercus es dominante en gran variedad de comunidades vegetales, tiene gran importancia ecológica dentro de éstas, pero todavía no cuenta con un número definido de especies en varias ANP (áreas naturales protegidas), como la Sierra Fría, en Aguascalientes. El objetivo del presente trabajo fue contribuir al conocimiento de encinos dentro de esta reserva. Los datos se obtuvieron por muestreos y análisis de ejemplares depositados en el herbario de la Universidad Autónoma de Aguascalientes (UAA). Se muestra una lista de las especies de encinos presentes en el área natural protegida de Sierra Fría, Aguascalientes, con un total de 21 especies del género Quercus para el área natural. De ellas, cuatro se distribuyen únicamente en esta zona de las descritas en el estado. Q. potosina y $Q$. eduardii fueron las especies con mayor distribución.

\section{ABSTRAC1}

The genus Quercus is dominant in a variety of plant

Palabras clave: Aguascalientes; bosque templado; diversidad; encinos; conservación.

Keywords: oaks; center of Mexico; temperate forest; diversity; botany; conservation.

\section{Recibido: 9 de junio de 2016, aceptado: 16 de diciembre de 2016}

* Departamento de Biología, Centro de Ciencias Básicas, Universidad Autónoma de Aguascalientes. Avenida Universidad 940, C. P. 20131, Aguascalientes, Aguascalientes, México. Correo electrónico: victor.mtz.calderon@gmail.com; masiquei@correo.vaa.mx; jmartinr@correo.vaa.mx

$凶$ ron@gmail.com; masiquei@cor para correspondencia communities, having great ecological importance within natural forest, but without a defined number of species in several NPAs such as the Sierra Fria, Aguascalientes. The goal of this study was to contribute to the knowledge of oaks within NPA, Sierra Fria. Data were obtained from samples and analysis of HUAA herbarium specimens. 21 species of the genus Quercus are present in Sierra Fria. Four of these species are distributed only in this area within the state. $Q$. potosina and $Q$. eduardii are the most widely distributed.

INTRODUCCIÓN

El género Quercus es de gran importancia ecológica porque sus especies son dominantes en gran variedad de comunidades vegetales (Nixon, 2006). En México, el número de especies es controversial, pero no hay un consenso acerca del número preciso por la dificultad al identificarlas (González Rivera, 1993; Rzedowski, 2006; Valencia, 2004; ZavalaChávez, 1998). De los dos subgéneros de Quercus, Cyclobalanopsis y Quercus, en México sólo está representado el segundo, con tres secciones: Quercus (encinos blancos) con 81 especies, Lobatae (encinos rojos) con 76, y Protobalanus (encinos intermedios 0 de copa dorada) con 4 de las 5 especies (Valencia, 2004). Muchas de las especies de encinos son preservadas en las distintas áreas naturales protegidas a lo largo de nuestro país.

Las áreas naturales protegidas (ANP) han sido importantes dentro del país en la conservación de la biodiversidad, así como en la prestación de servicios 


\section{IIVESTIGAGIÓn Y CUERCIA DE LA UกIVERSIDAD AUTÓOOOMA DE RGUASCALIENTES}

ecológicos y ecoturísticos. En el estado de Aguascalientes hay zonas prioritarias por su importancia ecológica o cultural; es decir, áreas naturales protegidas, como el monumento ecológico Cerro del Muerto, el cerro Juan el Grande y cerro De los Gallos, Sierra de Laurel y Sierra Fría (SF), esta última la de mayor extensión e importancia dentro del estado.

Se han reportado en diversos trabajos las especies presentes en el estado de Aguascalientes (De la Cerda, 1989, 1999; McVaugh, 1974). Sin embargo, en particular para la Sierra Fría no se tenía un listado exacto de encinos presentes en la zona, a excepción del reportado por Díaz, Sosa-Ramírez y Pérez-Salicrup (2012) y Sosa-Ramírez, Moreno-Rico, Sánchez-Martínez, Siqueiros-Delgado y Díaz-Núñez (2011), quienes mencionan para esta área, 10 y 11 especies, respectivamente. El presente estudio tuvo como objetivo dar a conocer las especies de encinos presentes en la Sierra Fría y aumentar el conocimiento del género en dicha ANP.

\section{MATERIALES Y MÉTODOS}

\section{Área de estudio}

El área natural protegida de Sierra Fría cubre una superficie de 112,000 ha, se ubica al noroeste del es-

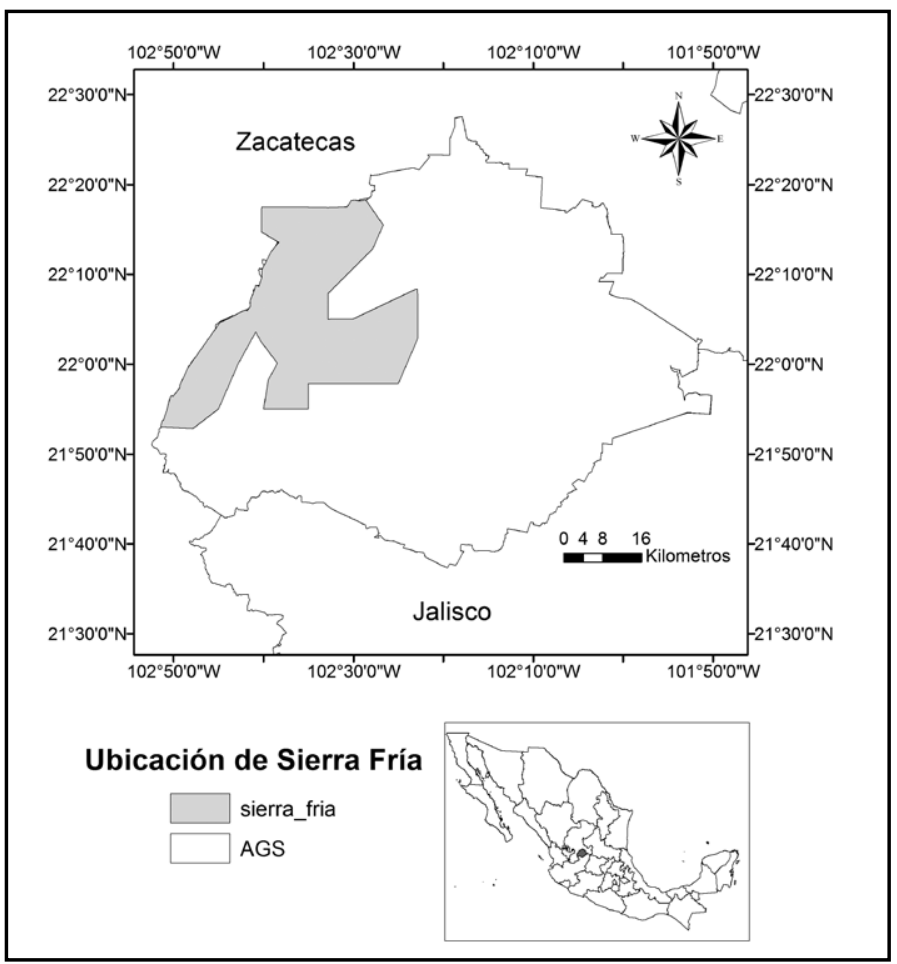

Figura 1. Ubicación de Sierra Fría dentro del estado de Aguascalientes y estados colindantes.

Elaboración propia.

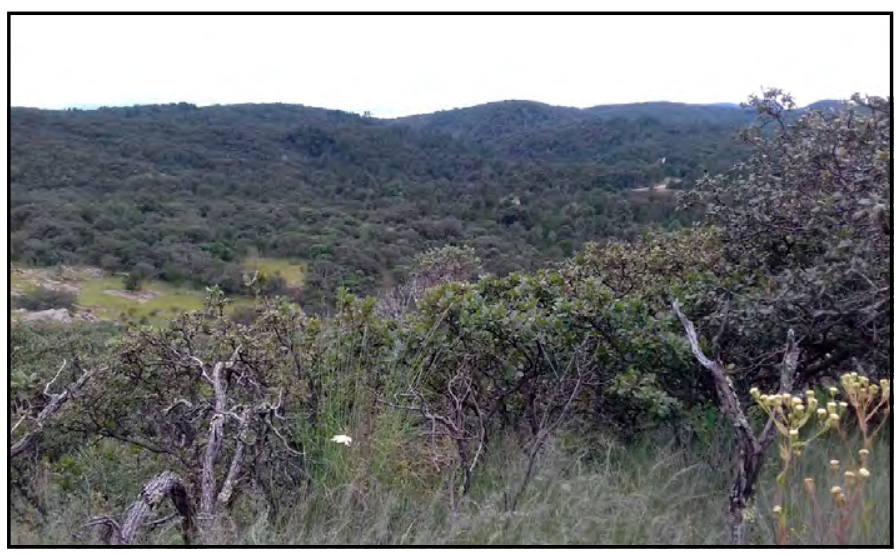

Figura 2. Bosque de encino en Sierra Fría, Aguascalientes.

Fotografía proporcionada por los autores.

tado de Aguascalientes, formando parte de la Sierra Madre Occidental (figura 1), en una altitud que oscila entre los 2,100 y los 3,050 m.s.n.m. (Díaz et al., 2012). Los climas predominantes son templados y semiáridos templados; su temperatura media anual varía entre 12.0 y $18.0^{\circ} \mathrm{C}$, la temperatura media del mes más frio oscila entre -3.0 y $18.0^{\circ} \mathrm{C}$ y la precipitación total anual es de 600 a $800 \mathrm{~mm}$. El tipo de vegetación predominante es el bosque de encino-pino, encino y encino-táscate (Juniperus deppeana). La asociación existe con pinos por arriba de los 2,450 $m$ y táscate por debajo de esta altitud. También existe matorral templado, árido y subtropical; matorral crasicaule, espinoso, rosetófilo; chaparrales y pastizal natural. Los encinares (figura 2) son los mejor conservados con un importante número de especies (Arriaga et al., 2000).

\section{Material biológico}

Se realizaron recolectas de enero a noviembre de 2014 en puntos previamente establecidos en las regiones de vegetación natural del ANP Sierra Fría. Se obtuvieron al menos tres muestras representativas de cada especie con las estructuras necesarias para su identificación. En cada sitio de muestreo se tomaron datos de campo como altitud, coordenadas geográficas con base en Datum WGS 84 y tipo de vegetación. Además, se revisaron los ejemplares depositados en el herbario de la Universidad Autónoma de Aguascalientes (HUAA) y se recopiló información de estudios anteriores dentro de la zona de investigación (Díaz et al., 2012; Sosa-Ramírez et al., 2011).

La determinación de los ejemplares se llevó a cabo utilizando claves taxonómicas especializadas para el género (Espinosa, 2001; González Villarreal, 
IIVESTIGACIÓก Y CIECIGIA DE LA UNIVERSIDAD AUTÓNOMA DE RGUASCALIERTES
1986; McVaugh, 1974; Sabás-Rosales, 2011). Con base en los datos tomados de campo y en los ejemplares del herbario se realizaron mapas de distribución con SIG, mediante un corte de las coordenadas obtenidas de recolectas y ejemplares del HUAA sobre el polígono del ANP Sierra Fría, con el fin de conocer la totalidad de especies que se distribuyen en dicha reserva (figuras 3 y 4). Se buscaron las coordenadas de las localidades de examinados en el HUAA, en caso de que no estuvieran disponibles en la etiqueta. Los ejemplares recolectados fueron depositados en el mismo herbario.
Dentro del ANP Sierra Fría se distribuyen 21 especies del género Quercus, 4 de ellas exclusivas de esta zona (tabla 1, figuras 3 y 4 ), las que se encuentran principalmente en bosque de encino y bosque mixto, a una altitud promedio de 2,200-2,800 m. Las especies más ampliamente distribuidas son $Q$. potosina y $Q$. eduardii. En trabajos anteriores (Díaz et al., 2012; Sosa Ramírez et al., 2011) no se tenían mencionadas 10 especies para el ANP Sierra Fría: Q. castanea Née, Q. depressipes Trel., Q. durifolia Seemen, Q. gentry C. H. Müll., Q. greggii (A.DC.) Trel., Q. laurina Humb. \& Bonpl., Q. magnoliifolia Née, Q. mexicana Humb. \& Bonpl., Q. repanda Humb. \& Bonpl., y Q. striaturla Trel.

Tabla 1

Especies de encino (Quercus) en SF, Aguascalientes, México

\begin{tabular}{|c|c|c|}
\hline Sección/ Taxón & Altitud & Tipo de vegetación \\
\hline \multicolumn{3}{|l|}{ Lobatae (encinos rojos) } \\
\hline Q. aristata Hook. \& Arn. & $1,640-2,600$ & $B Q$ \\
\hline Q. castanea Née* & $2,550-2,695$ & $B Q$ y $B M$ \\
\hline Q. durifolia Seemen*+ & $2,400-2,839$ & $B Q$ y $B M$ \\
\hline Q. eduardii Trel. & $2,050-2,800$ & $B Q, B M, P T$ \\
\hline Q. gentry C.H. Müll.* & $2,200-2,450$ & $B Q$ \\
\hline Q. jonesii Trel. & $2,450-2,800$ & $B Q$ y $B M$ \\
\hline Q. laurina Humb. \& Bonpl.*+ & 2,839 & BM \\
\hline Q. mexicana Humb. \& Bonpl..+ & 2,112 & $B Q$ \\
\hline Q. sideroxyla Humb. \& Bonpl. & $2,300-2,900$ & $B Q$ y $B M$ \\
\hline \multicolumn{3}{|l|}{ Quercus (encinos blancos) } \\
\hline Q. chihuahuensis Trel. & $1,950-2,800$ & $B Q$ y $P T$ \\
\hline Q. depressipes Trel.*+ & $2,177-2,244$ & $B Q$ y $P T$ \\
\hline Q. greggii (A. DC.) Trel. & 2,621 & $\mathrm{BM}$ \\
\hline Q. grisea Liebm. & $1,900-2,600$ & $B Q, B M, M T$ y $P T$ \\
\hline Q. laeta Liebm. & $2,050-2,800$ & $B Q, B M y P T$ \\
\hline Q. magnoliifolia Née* & 2,541 & $B Q$ \\
\hline Q. obtusata Humb. \& Bonpl. & $2,100-2,700$ & $B Q$ \\
\hline Q. potosina Trel. & $2,100-2,800$ & $B C, B Q, B M, M T$ y $P T$ \\
\hline Q. repanda Humb. \& Bonpl.* & $2,250-2,750$ & $B Q, B M, B C$ y $P T$ \\
\hline Q. resinosa Liebm. & $2,160-2,600$ & $B Q, B Q-P T$ \\
\hline Q. rugosa Née & $2,100-2,800$ & $B Q, B M$ y $B Q-M T$ \\
\hline Q. striatula Trel. & 2,574 & $\mathrm{BM}$ \\
\hline
\end{tabular}

Nota: $\left({ }^{*}\right)$ Especie no mencionada anteriormente para SF, Aguascalientes. (+) Exclusivo de SF dentro del estado. bosque de encino (BQ), bosque mixto (BM), pastizal templado (PT), matorral templado (MT), bosque de coníferas (BC). Elaboración propia. 


\section{IIVESTIGAGIÓn Y CUERCIA DE LA UחIVERSIDAD AUTÓNOMA DE RGUASCALIENTES}

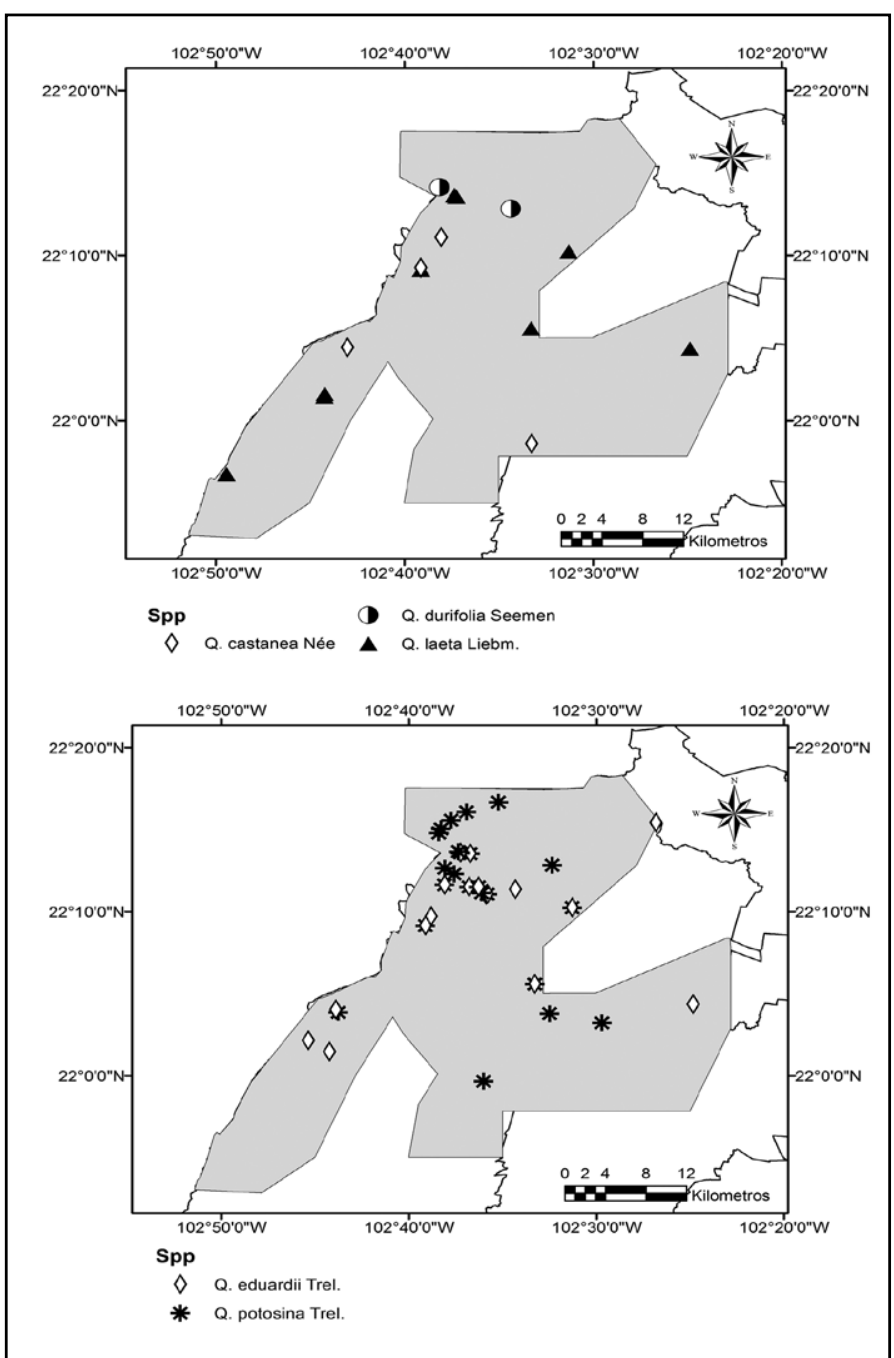

Figura 3. Puntos de recolecta de las especies de encinos en Sierra Fría, Aguascalientes, basados en recolectas y ejemplares del HUAA. Elaboración propia.

\section{DISCUSIÓN}

El ANP Sierra Fría cuenta con $13 \%$ de especies de México: 161 especies (Valencia, 2004). Si bien ninguna de las especies encontradas dentro de la zona de estudio se encuentra en peligro, la riqueza de especies es alta comparada con otros sitios; por ejemplo, en el Cerro del Águila, Michoacán, se encuentran 7 especies de encinos (Zacarías-Eslava, CortejoTenorio, Cortés-Flores, González-Castañeda, \& IbarraManríquez, 2011), Sierra de Santa Rosa, Guanajuato, cuenta con 8 encinos (Martínez- Cruz, Téllez-Valdés, \& Ibarra-Manríquez, 2009) y la Reserva de la Biósfera Mariposa Monarca, que alberga 8 especies de encinos, los tres sitios con un número pequeño de

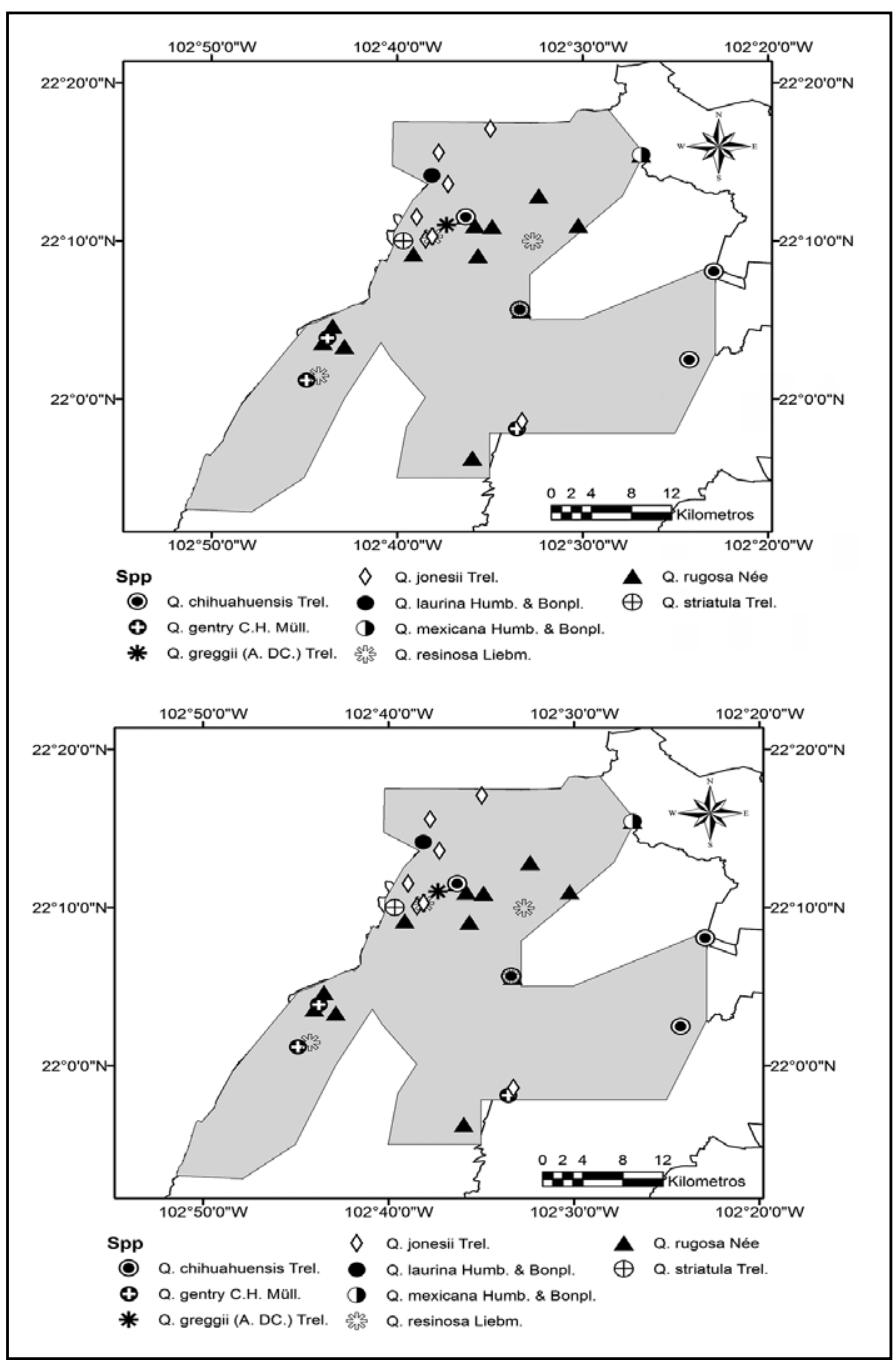

Figura 4. Puntos de recolecta de las especies de encinos en Sierra Fría, Aguascalientes, basados en búsquedas y ejemplares del HUAA. Elaboración propia.

especies comparado con Sierra Fría. También existen sitios similares en cuanto a riqueza como la Reserva de la Biósfera Sierra de Manantlán, con una superficie de 137,000 ha, alberga 31 especies de encinos (OlveraVargas, Figueroa-Rangel, Vázquez-López, \& Brown, 2006), al compararse con la superficie de Sierra Fría de 112,000 ha, que alberga 21 especies de encinos.

México es un centro de diversificación de este género, su endemismo es importante; Valencia (2004) menciona que existen 109 especies endémicas de encinos mexicanos que equivalen a $67.7 \%$ del total de especies del género en México. De las especies encontradas sólo $Q$. castanea, $Q$. chihuahuensis, $Q$. depressipes, Q. grisea, Q. rugosa se distribuyen fuera 
IIVESTIGACIÓn Y CIERCIA DE LA UNIVERSIDAD AUTÓNOMA de los límites del país, en donde 16 especies de Sierra Fría son endémicas de México y representan $14.6 \%$ de las 109 especies.

En el ANP de Sierra Fría se encuentra $84 \%$ de la riqueza de encinos en Aguascalientes, encontrándose 21 de las 25 especies mencionadas para el estado. Solo Q. candians, Q. desertícola, Q. praeco y $Q$. viminea no se llegan a encontrar dentro del ANP (Martínez-Calderón, Siqueiros-Delgado, \& MartínezRamírez, 2017).

Especies como Q. depressipes, Q. greggii, Q. repanda y $Q$. striatula son encinos de porte arbustivo distribuidos del ANP. Q. repanda y $Q$. striatula presentan crecimiento rizomatoso, se llegan a encontrar formando parte del sotobosque en bosque de encino y bosque mixto, con mayor frecuencia se llega a encontrar $Q$. repanda, siendo más frecuente que $Q$. striatula. Q. depressipes, distribuido de los 2,177 a los 2,244 m, se llega a encontrar en partes altas y expuestas, donde este encino tiene buenas poblaciones, junto con Q. potosina. Se encontró por primera vez en la estación biológica Agua Zarca, perteneciente a la UAA; siendo parte del ANP Sierra Fría, se registró como parte de un informe florístico de la estación. Posteriormente se reporta la presencia de Q. obtusata para el ANP por Díaz et al. (2012) como parte de un estudio de distribución de especies forestales; esta especie puede encontrarse en sitios húmedos, en las partes altas de Sierra Fría, junto con Q. sideroxyla y Q. rugosa.

En el ANP de Sierra Fría se presenta con mayor frecuencia $Q$. potosina, y en orden descendiente $Q$. eduardii, Q. sideroxyla, Q. grisea y Q. rugosa (SosaRamírez et al., 2011) y son encontradas en amplio rango altitudinal y de tipo de vegetación (tabla 1.). Por el contrario, existen especies cuya distribución está restringida (Q. aristata, Q. durifolia, Q. greggii, $Q$. laurina). La presencia de especies de encinos está ligada a condiciones particulares de altitud y tipo de vegetación, al igual que a factores de humedad, tipo de suelos, o exposición solar. Las especies de encinos rojos están mayormente restringidos a sitios húmedos dentro de Sierra Fría, como Q. sideroxyla y $Q$. eduardii: se localizan en sitios con exposiciones norte, en laderas medias y bajas, en altitudes promedio de 2,050 a $2,800 \mathrm{~m}$ para $Q$. eduardii, de 2,300 a 2,900 m para $Q$. sideroxyla donde en altitudes grandes llega a presentar gran abundancia, y coberturas de dosel superiores a 50\% (Sosa-Ramírez et al., 2011), en donde Q. eduardii es el encino rojo con mayor distribución dentro del ANP.

Quercus jonesii se encuentra en partes altas de la Sierra Fría de 2,700 a 2,900 m, en bosque de encino-pino, acompañado de Pinus lumholtzii B. L. Rob. \& Fernald, sobre laderas pedregosas y calizas (De la Cerda, 1999; Sosa-Ramírez et al., 201 1), en este tipo de asociación pino-encino, $Q$. jonesii suele ser abundante, aunque esta asociación vegetal es de distribución restringida. Encinos como Q. durifolia, recolectado a una altitud de 2,400 y $2,800 \mathrm{~m}, \mathrm{Q}$. laurina $(2,800 \mathrm{~m})$ y $Q$. aristata $(2,500-2,600 \mathrm{~m})$ son escasos y de distribución restringida, se encuentran en fondo de cañadas húmedas, junto a $Q$. rugosa en bosque de encino y $Q$. aristata en bosque de encino-pino. Q. durifolia es mencionado por Romero-Rangel, Rojas-Zenteno y Rubio-Licona (2015) como una especie con problemas de supervivencia, esta especie fue recolectada únicamente en dos localidades dentro del ANP. Q. mexicana se encontró en bosque de encino en barrancas húmedas a una altitud de 2,112 m, asociado a Q. rugosa y Q. grisea, pero puede llegar a confundirse con ejemplares de Q. eduardii que presenten margen entero.

Q. gentry se ubica en altitudes de 2,200 a 2,450 m.s.n.m., en bosque de encino en partes sur de Sierra Fría, dentro del municipio de Calvillo y Jesús María, se llega a encontrar con Q. castanea, en lugares con laderas húmedas y pendientes medias. Q. castanea, distribuido a altitudes de 2,550 a 2,695, se encuentra formando parte de bosque de encino y bosque mixto, se puede encontrar como árboles de $10 \mathrm{~m}$ en Laguna Seca, localidad de Sierra Fría. Las especies de encinos blancos se llegan a encontrar en lugares más áridos, por ejemplo, Q. chihuahuensis, una de las especies que prefiere climas áridos dentro del ANP, se encuentra en pequeños grupos aislados, a menudo como vestigios de bosque de encino, sobre barrancas secas de la Sierra Fría.

Otra especie con resistencia a condiciones secas es $Q$. potosina, la especie con mayor distribución y abundancia dentro del ANP (Díaz et al., 2012; Sosa-Ramírez et al., 2011), se puede llegar a encontrar formando parte del boque mixto junto a J. deppeana y formando parte de matorrales junto a Arctostaphylos pungens, su distribución altitudinal se encuentra de 2,100-2,800 m. Q. grisea es otra especie que llega a formar asociaciones con J. deppeana y se llega a encontrar en lugares de poca humedad y con pendientes bajas. Al contrario de Q. rugosa, que prefiere sitios de humedad, se 


\section{IIVESTIGAGIÓn Y CUERCIA DE LA UחIVERSIDAD AUTÓNOMA DE AGUASCALIERTES}

distribuye entre los 2,100 y los 2,800 m, en lugares con pendientes medias y ligeras, en exposiciones norte y con fisiografías en bajo de ladera (Sosa-Ramírez et al., 2011), mayormente en bosque de encino. Mientras $Q$. resinosa tiene poca distribución en Sierra Fría, desde los 2,160 hasta los 2,600 m, normalmente es abundante en otras partes del estado, se ubica en partes bajas de las serranías y sitios de poca humedad, llega a ser de los primeros encinos en ser observado subiendo a la localidad de La Congoja, Sierra Fría, junto a Q. potosina y Q. eduardii.

Otra especie escasa en el ANP Sierra Fría y con mucha similitud morfológica a $Q$. resinosa es $Q$. magnoliifolia, que se localiza a una altitud de 2,541 $\mathrm{m}$, en bosque de encino y en la parte sur de la zona de estudio, en asociada a otros encinos como $Q$. potosina y $Q$. sideroxyla. Q. laeta, presenta gran polimorfismo foliar, lo cual dificulta su identificación en campo. Sosa-Ramírez et al. (2011) mencionan una distribución entre los 2,600-2,700 m dentro del ANP; sin embargo, en herbario se observaron ejemplares desde los 2,050 m. Dentro del ANP esta especie no es tan abundante como en el resto del estado, se llega encontrar en pendientes ligeras o planas, y en exposiciones sur dentro de Agua Zarca.

\section{CONCLUSIONES}

El total de especies encontradas en el ANP Sierra Fría representa $84 \%$ de la diversidad de encinos en Aguascalientes, solo $16 \%$ no se han encontrado ahí; esto demuestra la importancia que tiene esta ANP para la conservación de la biodiversidad del estado de Aguascalientes y la necesidad de realizar investigaciones en la zona, así como implementar planes de conservación.

REFERENCIAS

- Arriaga, L., Espinoza, J. M., Aguilar, C., Martínez, E., Gómez, L., \& Loa, E. (Coords.). (2000). Regiones terrestres prioritarias de México. México: Comisión Nacional para el Conocimiento y uso de la Biodiversidad.

- Comisión Nacional de Áreas Naturales Protegidas. (2011). Programa de Manejo Reserva de la Biósfera Mariposa Monarca, México (pp. 122). México: Autor.

- De la Cerda, L. M. (1989). Encinos de Aguascalientes. Aguascalientes, México: Universidad Autónoma de Aguascalientes.

(1999). Encinos de Aguascalientes. (2 ${ }^{\text {da }}$ ed.). México: Universidad Autónoma de Aguascalientes.

- Díaz, V., Sosa-Ramírez, J., \& Pérez-Salicrup, D. R. (2012). Distribución y abundancia de las especies arbóreas y arbustivas en la Sierra Fría, Aguascalientes, México. Polibotánica, 34, 99126.

- Espinosa, G. J. (2001). Fagaceae. En G. C. de Rzedowski, \& J. Rzedowski. Flora fanerogámica del Valle de México (2a . ed.) (pp. 81-91). México: CONABIO.

- González Rivera, R. (1993). La diversidad de los encinos mexicanos. Revista de la Sociedad Mexicana de Historia Natural, 44, 125-142.

- González Villarreal, L. M. (1986). Contribución al conocimiento del género Quercus (Fagaceae) en el estado de Jalisco. Colección Flora de Jalisco. Guadalajara, México: Universidad de Guadalajara.
Martínez-Calderón, V. M., Siqueiros-Delgado, M. E., \& MartínezRamírez, J. (2017). Checklist of the genus Quercus (Fagaceae) of Aguascalientes, Mexico. Check List, 13(1), 2045. doi: 10.15560/13.1.2045

- Martínez-Cruz, J., Téllez-Valdés, O., \& Ibarra-Manríquez, G. (2009). Estructura de los encinares de la sierra de Santa Rosa, Guanajuato, México. Revista Mexicana de Biodiversidad, $80(1), 145-156$.

- McVaugh, R. (1974). Flora Novo-Galiciana (Quercus). Contributions from the University of Michigan Herbarium, Ann Arbor, Michigan. 12, parte 1 (3).

- Nixon, K. C. (2006). Global and neotropical distribution and diversity of oak (genus Quercus) and oak forests. En M. Kappelle (Ed.), Ecology and conservation of neotropical montane oak forests (pp. 3-11). Germany: Springer-Verlag Berlin Heidelberg.

- Olvera-Vargas, M., Figueroa-Rangel, B. L., Vázquez-López, J. M., \& Brown, N. (2006). Dynamics and silviculture of montane mixed oak forest in western Mexico. En M. M. Caldwell et al. (Eds. de la serie), Ecological Studies: Vol. 185. Ecology and conservation of neotropical montane oak forest (pp. 363-374). Springer. doi: 10.1007/3-540-28909-7_28

- Romero-Rangel, S., Rojas-Zenteno, E. C., \& Rubio-Licona, E. (2015). Encinos de México. México: Universidad Nacional Autónoma de México.

- Rzedowski, J. (2006). Bosque de Quercus. En Vegetación de México (pp. 274-294). México: Comisión Nacional para el Conocimiento y Uso de la Biodiversidad. 
- Sabás-Rosales, J. L. (2011). Taxonomía, diversidad y distribución de los encinos (Quercus spp.) del estado de San Luis Potosí, México. Tesis de Maestría. Aguascalientes, México: Universidad Autónoma de Aguascalientes.

- Sosa-Ramírez, J., Moreno-Rico, O., Sánchez-Martínez, G., Siqueiros-Delgado, M. E., \& Díaz-Núñez, V. (2011). Ecología y fitosanidad de los encinos (Quercus spp.) en la Sierra Fría, Aguascalientes, México. Madera y Bosques, 17(3), 49-63.

- Valencia A., S. (2004). Diversidad del género Quercus (Fagaceae) en México. Boletín de la Sociedad Botánica de México, 75, 33-53.
- Zacarías-Eslava, L. E., Cortejo-Tenorio, G., Cortés-Flores, J., González-Castañeda N., \& Ibarra-Manríquez, G. (2011). Composición, estructura y diversidad del cerro El Águila, Michoacán, México. Revista Mexicana de Biodiversidad, 82, 854-869.

- Zavala-Chávez, F. (1998). Observaciones sobre la distribución de encinos en México. Polibotánica, 8, 47-64. 